\title{
Analysis of Prognostic Factors of Nasopharyngeal Carcinoma and Construction of Risk Nomogram Prediction Model
}

\section{xuan hao zhang ( $D$ 0100197@bbmc.edu.cn )}

Department of Human Anatomy, Bengbu Medical College, Anhui, China

\section{LU Jin}

Department of Human Anatomy, Bengbu Medical College, Anhui, China

JIANG Cheng-yi

First Affiliated Hospital of Bengbu Medical College, Anhui, China

\section{FANG Mei-fang}

School of Humanities and Management, Wannan Medical College, Anhui, China

\section{Liu Hai-rong}

Department of Human Anatomy, Bengbu Medical College, Anhui, China

\section{Research}

Keywords: nasopharyngeal carcinoma, predictive model, nomogram, risk factors

Posted Date: June 30th, 2021

DOl: https://doi.org/10.21203/rs.3.rs-645804/v1

License: (9) This work is licensed under a Creative Commons Attribution 4.0 International License. Read Full License 


\section{Abstract}

Objective To analyze the factors affecting the prognosis of nasopharyngeal carcinoma (NPC) patients and establish a risk nomogram prediction model for the diagnosis, treatment and prognosis of NPC.

Methods A total of 1645 NPC patients (1470 survival) from 1973 to 2015 in the SEER database were included. The clinicopathological data of 180 NPC patients hospitalized in the First Affiliated Hospital of Bengbu Medical College since 2010 were collected. The spearman correlation analysis and COX regression analysis were used to identify the prognostic factors of NPC and establish the risk nomogram prediction model. Receiver operation characteristic (ROC) analysis and area under curves (AUC) were used to evaluate the ability of distinguishing the prognosis of NPC patients. The cross calibration was calculated to evaluate the external validation of established predictive model.

Results The survival NPC had higher proportion in female, older age, degree of NPC differentiation, N and $M$ stage (all $P<0.05$. The study factors including female, older age, and higher degree of differentiation were positively correlated with the prognosis of NPC patients $(P<0.05)$, while $N$ and $M$ stage were negatively correlated with the prognosis of NPC patients $(P<0.05)$. The factors including female $(\mathrm{HR}=4.92,95 \% \mathrm{Cl} 3.58-6.75)$, age $(\mathrm{HR}=, 95 \% \mathrm{Cl}=)$, degree of differentiation $(\mathrm{HR}=, 95 \% \mathrm{Cl}=), \mathrm{N}$ stage $(\mathrm{HR}=$, $95 \% \mathrm{Cl}=)$ were independently associated with prognosis of NPC patients $(\mathrm{P}<0.05)$. Comparing with calibration curve, the constructed risk prediction model had good relations between predictive values and actual values $(A U C=0.833)$. Additionally, the external verification results of 96 patients with NPC had a larger AUC in our established predictive model $(A U C=0.851)$.

Conclusions Female, degree of differentiation and $\mathrm{N}$ stage are independent factors for the prognosis of NPC patients. The risk nomogram prediction model has a good predictive ability for the survival of NPC patients, which can provide important insights into clinical prognosis for NPC patients.

\section{Introuction}

Nasopharyngeal carcinoma (NPC) is a malignant tumor that occurs frequently in the roof and sidewall of the human nasopharynx. NPC has the highest incidence among malignant tumors of the ear, nose, and throat, which is also relatively specific malignant tumor in China. Globally, around $80 \%$ of NPC patients have been reported in southern China, mainly in Guangdong, Guangxi, and Hunan Province[1-2]. Due to the particularity of NPC anatomical location, the early clinical symptoms of NPC patients are asymptomatic. Approximate $\mathrm{x} \%$ ? NPC patients have been underdiagnosed at an advanced stage, without optimal the clinical treatment effect, resulting in a low 5-year overall survival (OS). However, the clinical cure rate of NPC with early diagnosis can approach 90\% [3-4]. In addition, the recurrence rate and metastasis rate of patients with advanced NPC are relatively high, which has been considered the main reason for the failure of clinical treatment [5]. Although the great progress of detection and radiotherapy techniques in NPC have been made recently, the mortality rate of NPC patients is still high. Therefore, it is of great practical significance to improve assessment of the prognosis in NPC patients. 
To date, the risk nomogram prediction model has been widely used in assessing risk for the prognosis of various tumor patients, with high accuracy and consistency [6-7]. Therefore, this study aims to analyzes the clinical data of patients with NPC and screens out the factors related to the prognosis of NPC in SEER database. Meanwhile, this study using clinical data form hospitalized NPC patients to establish a risk prediction model for the prognosis of NPC, and the investigate the validation of predictive model.

\section{Materials And Methods}

\subsection{Data source}

The modeling was established from the clinical data of NPC stored in the SEER database from 1973 to 2015 (including gender, age, degree of differentiation, TNM staging and survival status). The verification data were from the First Affiliated Hospital of Bengbu Medical College since $2010(n=180)$ Clinical data of each hospitalized patient (including gender, age, degree of differentiation, TNM staging and metastasis status).

\subsection{Inclusion and exclusion criteria}

Data inclusion criteria (1) pathologically diagnosed as NPC; (2) completed clinical and pathological data; (3) no history of radiotherapy and chemotherapy.

Data exclusion criteria (1) non-nasopharyngeal cancer; (2) severe lack of clinical and pathological data; (3) history of radiotherapy and chemotherapy. A total of 1645 cases were selected in SEER database for modeling establishing and 96 cases were selected as clinically verified data.

\subsection{Statistical methods}

The chi-square performing in SPSS 19.0 was used to compare observsed size between groups (by sex, age, degree of differentiation, and stage $T, N, M$ ). The multivariable COX regression was used to analyze the risk factors for NPC prognosis. The R was used to establish nomograph model, calibration curve and ROC. The consistency and applicability of established model was calculated. A two-tail $p$-value $<0.05$ was considered statistical significance.

\section{Results}

2.1 Baseline data and clinicopathological characteristics of patients with nasopharyngeal carcinoma The characteristics of 1645 cases (1470 surviving, female ? \%) of NPC from SEER database are presented in Table 1 and Table 2. the majority age range from 40 to 80 years. Most of them (?\%) were moderately or poorly differentiated. Pervcentage of $x x x$ between gender, age, and degree of differentiation had significant differences $(\mathrm{P}<0.05)$. Regarding the TNM staging, $\mathrm{T} 1 \mathrm{?} \%, \mathrm{~N} 1 \mathrm{?} \%$, and $\mathrm{M} 0$ ?\% are the most. Except for the T-stage, differences between $\mathrm{N}$-stage and $\mathrm{M}$-stage groups were statistically significant $(P<0.05)$. 
2.2 Correlation of clinicopathological characteristics and prognosis of patients with nasopharyngeal carcinoma

There were positive correlations between gender (female), age, and degree of differentiation with the prognosis of NPC $(P<0.05)$. Except The T stage, TNM staging was negatively correlated with the prognosis of NPC. Additionally, both N stage and M stage had significantly negative correlations with prognosis of $\mathrm{NOC}(\mathrm{P}<0.05)$.

The results of univariate COX regression analysis are shown in Table 4.In univariable cox regression, Except for $\mathrm{T}$ stage gender, age, degree of differentiation, $\mathrm{N}$ stage and $\mathrm{M}$ stage were significantly associated with prognosis of NPC (all P<0.05). After adjustment for ???, gender, degree of differentiation and $\mathrm{N}$ stage remained significant associations with the prognosis of NPC (all P<0.001). Therefore, gender, degree of differentiation and $\mathrm{N}$ stage were considered independent risk factors for the prognosis of NPC.

\subsection{Construction and evaluation of the prediction model of nasopharyngeal carcinoma risk nomogram}

Based on the modeling data of NPC in the SEER database, the characteristics (including gender, age, degree of differentiation, and TNM staging) of the clinical data were included into the prognostic risk nomogram model for constructing the prognosis of NPC. The results showed that tumor staging (TNM) had greater impact on the prognosis of NPC, while the age had smaller impact(Figure 1). The calibration curves showed a good consistency between the predicted value and the true observed values (Figure 2). Meanwhile, ROC curve analysis was performed in the modeling data. The results showed that the area under the curve (AUC) was 0.833 (Figure 3). Therefore, the risk nomogram prediction model has good discriminative utility, as citation

\subsection{External verification of the prediction model of nasopharyngeal cancer risk nomogram}

The clinical data of 96 patients with NPC with distant metastasis from the hospital were collected as external verification data. The probability of distant metastasis of this group of patients with nasopharyngeal carcinoma was $55.2 \%$. The specific cases information was shown in Table 5. Based on the verification data, the collected clinical data of NPC patients with distant metastasis was verified for consistency with the prediction model. The results in calibration curve showed a better consistency between the predicted value and the true value (Figure 4).Meanwhile, the AUC of the verification data was 0.851 (Figure 5). Therefore, this model has high feasibility for external data applications.

\section{Discussion}

Summary of main findings in this study

At present, the prognosis assessment method of nasopharyngeal carcinoma is mainly based on TNM staging, but TNM staging only involves tumor expression load, and does not take into account the impact of tumor cells on the prognosis of nasopharyngeal cancer patients, which causes errors in the treatment 
and prognosis of patients [8] . The risk nomogram is based on the patient's tumor pathological data and individual characteristics to determine the disease risk. It is a new type of statistical prediction model. Compared with the previous prognostic evaluation methods, it shows very high predictive power and has been widely used. Applied to the clinic [9]. In addition, the risk nomogram can also be used to evaluate tumor cell metastasis and recurrence, the effect of adjuvant therapy, and various survival prognosis [1012].

Although there have been few reports on the application of risk nomograms in the prognostic evaluation of patients with nasopharyngeal carcinoma, studies based on SEER database modeling and collecting clinical data for external verification have not been reported. Therefore, this article conducts an in-depth study on this in order to provide a basis for the diagnosis, treatment and prognosis of patients with clinical nasopharyngeal carcinoma.

This study analyzed the clinical data of nasopharyngeal carcinoma patients in the SEER database indicated that gender, tumor cell differentiation and $\mathrm{N}$ stage were independent risk factors for the prognosis of NPC. Previous studies in China have reported that the incidence and mortality of men with nasopharyngeal cancer were 2.4-3 times higher than women[13]. Another study analyzed the clinical data of 337 patients with recurrent nasopharyngeal carcinoma and indicated that male nasopharyngeal carcinoma patients had significantly higher probability of recurrence and metastasis and worse prognosis than female[14]. In addition, the data of nasopharyngeal cancer patients registered in the "China Cancer Registry Annual Report" from 2005 to 2013 reported a significant correlation between the death of nasopharyngeal cancer patients and gender[15-16]. This study consistently found that men with nasopharyngeal cancer had more incidences than women. However, women with nasopharyngeal cancer had a higher risk of death than men. This difference may be related to fewer female patients in the collected cases. Furthermore, this data suggests that for a good survival prognosis, positive intervention should be conducted in women once diagnosed with nasopharyngeal carcinoma.

The degree of differentiation of nasopharyngeal carcinoma tissue cells is highly correlated to the prognosis of NPC. This study found that the high-differentiated and undifferentiated NPC patients had lower risk of prognosis? death or survival? than moderately and poorly differentiated NPC patients. Huang and colleagues ${ }^{[17]}$ using the clinicopathological data of 1286 NPC patients found that the degree of differentiation of NPC tumor cells was closely related to their biological behavior, and affected tumor staging, lymph node metastasis and treatment, which consequently led to various prognosis in NPC patients. In addition, other domestic and international studies found that the degree of differentiation of nasopharyngeal carcinoma tumor cells was closely related to the degree of malignancy, and affected the survival and prognosis of patients[18-20]. The higher the differentiation of nasopharyngeal carcinoma tumor cells had the better the prognosis of patients.. However, another study ${ }^{[21]}$ reported that the radiotherapy sensitivity of nasopharyngeal carcinoma tissue was positively correlated to the division activity of tumor cells but inversely correlated to the degree of differentiation in nasopharyngeal carcinoma patients after radiotherapy. Given this, poorly differentiated and undifferentiated nasopharyngeal carcinoma tissues were more sensitive to radiotherapy due to more active tumor cell 
division, while well-differentiated nasopharyngeal carcinoma tissues were less effective due to weak cell division activities. Therefore, this study suggests that patients with poorly differentiated and undifferentiated nasopharyngeal carcinoma could be actively treated with radiotherapy to improve the clinical prognosis.

The TNM staging played an important role in assessing the prognosis of cancer patients, but paucity of individualized factors. This study found that $\mathrm{N}$ stage was an independent factor for the prognosis of patients with nasopharyngeal carcinoma, suggesting a higher prognostic risk. Some researchers pointed that compared with $\mathrm{T}$ staging, $\mathrm{N}$ staging mainly affected the distant metastasis of patients with nasopharyngeal carcinoma[22-23]. Additionally, in the case of radiotherapy and platinum-based adjuvant treatment, local $\mathrm{N}$-advanced nasopharyngeal carcinoma ( The prognosis of patients with T1ヌ2N2 $₫ 3 \mathrm{M} 0$ ) had worse prognosis than that of local T stage $(T 3 \otimes 4 \mathrm{NO} 01 \mathrm{M} 0)^{[24]}$. Some clinical studies found that later $\mathrm{N}$ stage of nasopharyngeal carcinoma patients had higher the distant metastasis rate and the worse prognosis ${ }^{[22]}$. Hua Yijun ${ }^{[25]}$ and colleagues found that the 5-year survival rate of patients with nasopharyngeal carcinoma in stage N3 was significantly lower than that in stage N2. The risk of distant metastasis in patients with nasopharyngeal carcinoma increased with the increasing in $\mathrm{T}$ stage. The results were consistent with many other studies, which suggested that the accuracy of the constructed risk nomogram model. Meanwhile the $\mathrm{N}$ stage is an independent risk for prognosis of patients with nasopharyngeal carcinoma. Therefore, the use of systemic and stratified treatment of patients with locally advanced nasopharyngeal carcinoma might be advocated to improve the prognosis of patients.

Considering the consistency and applicability of the prediction model with external verification, this study also collected clinical data of nasopharyngeal carcinoma for comparative analysis. The external verification results showed that the predicted value of the risk nomogram prediction model had good consistency with the true value. And the AUC of the internal and external verification models were both greater than 0.8 suggesting the good applicability of prediction model in clinical. However, there may be some bias between the platform data and the collected clinical data, the limitations of the data cannot be ignored when interpreting the analysis results.

In conclusion, this study used the method of big data analysis to established nasopharyngeal cancer risk nomogram prediction model, which has the good predictive ability and has important clinical significance for assessing the prognosis of nasopharyngeal cancer patients with values of popularization and application in clinical.

\section{Conclusions}

-Female, degree of differentiation and $\mathrm{N}$ stage are independent factors for the prognosis of NPC patients.

-The risk nomogram prediction model has a good predictive ability for the survival of NPC patients, which can provide important insights into clinical prognosis for NPC patients. 


\section{Declarations}

\section{Acknowledgments}

We thank Fang Meifang of the First Affiliated Hospital of Bengbu Medical College for his contribution in case collection, and Liu Hairong of Southern Anhui Medical College for his guidance in language.

\section{Authors'contributions}

LU Jin drafted the first version of the manuscript and worked out literature research. ZHANG Hao-xuan worked out the study design. FANG Mei-fang, JIANG Cheng-yi and LIU Hai-rong coordinated the study.LU Jin and ZHANG Hao-xuan did the statistical analyses. All authors read and approved the final manuscript and made substantial contributions during the review process.

\section{Funding}

This work was partially supported by the Key Project of Natural Science Research of Education Department of Anhui Province(No.KJ2020A0553).

\section{Availability of data and materials}

The datasets used and/or analysed during the current study are available

from the corresponding author on reasonable request.

\section{Ethics approval and consent to participate}

This article does not report on any studies with patients or animals that were performed by any of the authors.

\section{Consent for publication}

applicable.

\section{Conflict of interest}

The authors have no conflicts of interest to declare.

\section{Author details}

${ }^{1}$ Department of Human Anatomy, Bengbu Medical College, Anhui, China

${ }^{2}$ Key Laboratory of Digital Medicine and Smart Health in Anhui Province, Bengbu Medical College, Anhui, China

${ }^{3}$ First Affiliated Hospital of Bengbu Medical College, Anhui, China 
${ }^{4}$ School of Humanities and Management, Wannan Medical College, Anhui, China

\section{References}

[1] Chen YP, Chan ATC, Le QT, et al. Nasopharyngeal carcinoma.[J]. Lancet. 2019,394(10192):64-80.

[2] Yang $Y$, Zhang $P$, Zhang $\mathrm{H}$, et al. Immunocyte infiltration characteristics of gene expression profile in nasopharyngeal carcinoma and clinical significance[J]. Xi Bao Yu Fen Zi Mian Yi Xue Za Zhi. 2020, 36(12):1069-1075.

[3] Ben-Ami T, Ash S, Ben-Harosh M, et al. Nasopharyngeal carcinoma in children and young adultsBeyond 5-year survival[J]. Pediatr Blood Cancer. 2020,67(9):e28494.

[4] Chen MC, Kuan FC, Huang SF, et al. Accelerated Risk of Premature Ischemic Stroke in 5-Year Survivors of Nasopharyngeal Carcinoma[J]. Oncologist. 2019,24(9):e891-e897.

[5] Ng WT, Corry J, Langendijk JA, et al. Current management of stage IV nasopharyngeal carcinoma without distant metastasis[J]. Cancer Treat Rev. 2020,85:101995.

[6] Bandini M, Fossati N, Briganti A. Nomograms in urologic oncology, advantages and disadvantages[J]. Curr Opin Urol. 2019,29(1):42-51.

[7] Hu C, Yang J, Huang Z, et al. Diagnostic and prognostic nomograms for bone metastasis in hepatocellular carcinoma[J]. BMC Cancer. 2020,20(1):494.

[8] Li J, Chen S, Peng S, et al. Prognostic nomogram for patients with Nasopharyngeal Carcinoma incorporating hematological biomarkers and clinical characteristics[J]. Int J Biol Sci. 2018,14(5):549-556.

[9] Klatte T, Rossi SH, Stewart GD. Prognostic factors and prognostic models for renal cell carcinoma: a literature review. World J Urol. 2018,36(12):1943-1952.

[10]Gaitanidis A, Machairas N, Alevizakos M, Tsalikidis C, Tsaroucha A, Pitiakoudis M. Predictive Nomograms for Synchronous Liver and Lung Metastasis in Colon Cancer[J]. J Gastrointest Cancer. 2020,51(3):925-931.

[11]Yan Y, Zhou Q, Zhang M, et al. Integrated Nomograms for Preoperative Prediction of Microvascular Invasion and Lymph Node Metastasis Risk in Hepatocellular Carcinoma Patients[J]. Ann Surg Oncol. 2020,27(5):1361-1371.

[12]Yan Y, Liu H, Mao K, et al. Novel nomograms to predict lymph node metastasis and liver metastasis in patients with early colon carcinoma[J]. J Transl Med. 2019,17(1):193.

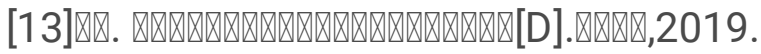




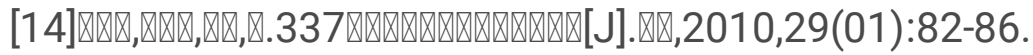

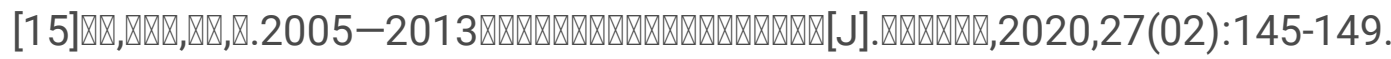

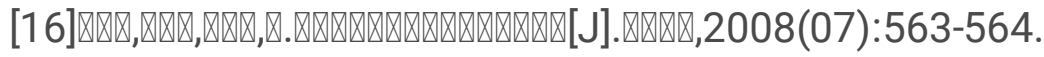

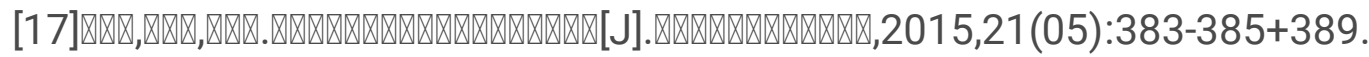

[18]Ren M, Wang Z, Gao G, Gu X, Wu L, Chen L. Impact of X-linked inhibitor of apoptosis protein on survival of nasopharyngeal carcinoma patients following radiotherapy[J]. Tumour Biol. 2016,37(9):11825-11833.

[19]Chen Y, Chen Z, Su Y, et al. Metabolic characteristics revealing cell differentiation of nasopharyngeal carcinoma by combining NMR spectroscopy with Raman spectroscopy[J]. Cancer Cell Int. 2019,19:37. [20]Wang J, Jiang Q, Faleti OD, et al. Exosomal Delivery of AntagomiRs Targeting Viral and Cellular MicroRNAs Synergistically Inhibits Cancer Angiogenesis [J]. Mol Ther Nucleic Acids. 2020,22:153-165.

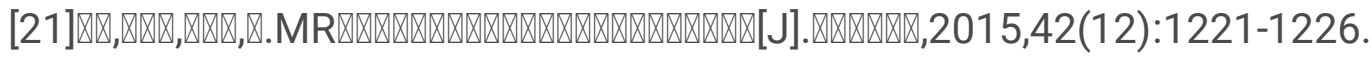

[22]Xu JH, Guo WJ, Bian XH, et al. A comparative study of locoregionallyadvanced nasopharyngeal carcinoma treated with intensity modulated irradiation and platinum-based chemotherapy[J]. Cancer Radiother,2013, 17(4):297-303.

[23]Zeng L, Tian YM, Sun XM, et al. Intensity-modulated radiotherapy for stage IVA/IVB nasopharyngeal carcinoma: clinical outcomes and patterns of failure in an endemic area in China[J]. Strahlenther Onkol,2014, 190(11):993-1000.

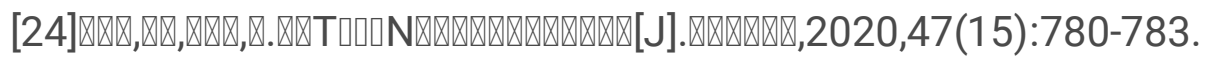

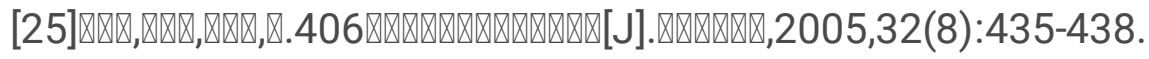

\section{Tables}

Table 1. Baseline data of nasopharyngeal carcinoma data in SEER database $(\mathrm{N}=1645)$ 


\begin{tabular}{|c|c|c|c|c|}
\hline \multicolumn{2}{|l|}{ Classify } & \multirow{2}{*}{$\begin{array}{l}\text { Code } \\
1\end{array}$} & \multirow{2}{*}{$\begin{array}{l}n \\
1116\end{array}$} & \multirow{2}{*}{$\begin{array}{l}\% \\
67.8\end{array}$} \\
\hline Sex & Male & & & \\
\hline & Female & 2 & 529 & 32.2 \\
\hline \multirow[t]{5}{*}{ Age } & $<20$ & 1 & 48 & 2.9 \\
\hline & $>=20,<40$ & 2 & 184 & 11.2 \\
\hline & $>=40,<60$ & 3 & 764 & 46.4 \\
\hline & $>=60,<80$ & 4 & 581 & 35.3 \\
\hline & $>=80$ & 5 & 68 & 4.1 \\
\hline \multirow[t]{4}{*}{ Degree } & No differentiation & 0 & 711 & 43.2 \\
\hline & Poorly differentiated & 1 & 697 & 42.4 \\
\hline & Moderate differentiation & 2 & 191 & 11.6 \\
\hline & Highly differentiated & 3 & 46 & 2.8 \\
\hline \multirow[t]{6}{*}{ Stage_T } & T0 & 0 & 3 & 0.2 \\
\hline & $\mathrm{T} 1$ & 1 & 587 & 35.7 \\
\hline & $\mathrm{T} 2$ & 2 & 324 & 19.7 \\
\hline & T3 & 3 & 313 & 19.0 \\
\hline & T4 & 4 & 342 & 20.8 \\
\hline & $\mathrm{TX}$ & 5 & 76 & 4.6 \\
\hline \multirow[t]{7}{*}{ Stage_N } & NO & 0 & 451 & 27.4 \\
\hline & N1 & 1 & 530 & 32.2 \\
\hline & N2 & 2 & 413 & 25.1 \\
\hline & N3a & 3 & 45 & 2.7 \\
\hline & N3b & 4 & 134 & 8.1 \\
\hline & N3NOS & 5 & 18 & 1.1 \\
\hline & $N X$ & 6 & 54 & 3.3 \\
\hline \multirow[t]{2}{*}{ Stage_M } & MO & 0 & 1549 & 94.2 \\
\hline & M1 & 1 & 96 & 5.8 \\
\hline \multirow[t]{2}{*}{ Status } & Alive & 0 & 1470 & 89.4 \\
\hline & Dead & 1 & 175 & 10.6 \\
\hline
\end{tabular}


Abbreviation:

Table 2. c2 analysis of nasopharyngeal carcinoma data in SEER database 


\begin{tabular}{|c|c|c|c|c|}
\hline & & Alive & Dead & $P$ \\
\hline \multirow[t]{2}{*}{ Sex } & Male & 1054 & 62 & \\
\hline & Female & 416 & 113 & $0 ?<0.001$ \\
\hline \multirow[t]{5}{*}{ Age } & $<20$ & 43 & 5 & \\
\hline & $>=20,<40$ & 164 & 20 & \\
\hline & $>=40,<60$ & 696 & 68 & \\
\hline & $>=60,<80$ & 516 & 65 & \\
\hline & $>=80$ & 51 & 17 & 0.02 \\
\hline \multirow[t]{4}{*}{ Degree } & No differentiation & 668 & 43 & \\
\hline & Poorly differentiated & 628 & 69 & \\
\hline & Moderate differentiation & 139 & 52 & \\
\hline & Highly differentiated & 35 & 11 & $0<0.001 ?$ \\
\hline \multirow[t]{6}{*}{ Stage_T } & T0 & 3 & 0 & \\
\hline & $\mathrm{T} 1$ & 524 & 63 & \\
\hline & $\mathrm{T} 2$ & 279 & 45 & \\
\hline & T3 & 276 & 37 & \\
\hline & $\mathrm{T} 4$ & 316 & 26 & \\
\hline & $\mathrm{TX}$ & 72 & 4 & 0.07 \\
\hline \multirow[t]{7}{*}{ Stage_N } & No & 346 & 105 & \\
\hline & N1 & 492 & 38 & \\
\hline & N2 & 393 & 20 & \\
\hline & N3a & 44 & 1 & \\
\hline & N3b & 124 & 10 & \\
\hline & N3NOS & 17 & 1 & \\
\hline & $N X$ & 54 & 0 & $<0.001 ? 0$ \\
\hline \multirow[t]{2}{*}{ Stage_M } & MO & 1374 & 175 & \\
\hline & M1 & 96 & 0 & $<0.0010$ \\
\hline
\end{tabular}

Abbreviation: 
Table 3. univariable or multivariable Correlation analysis between nasopharyngeal carcinoma data in SEER database and prognosis of patients

\begin{tabular}{|lll|}
\hline Classify & coefficients & $P$ \\
Sex & 0.239 & 0 \\
\hline Age & 0.054 & 0.029 \\
\hline Degree & 0.196 & 0 \\
\hline Stage_T & -0.045 & 0.069 \\
\hline Stage_N & -0.181 & 0 \\
\hline Stage_M & -0.086 & 0 \\
\hline
\end{tabular}

Adjustment??

Table 4. COX regression analysis of nasopharyngeal carcinoma data in SEER database

\begin{tabular}{|lllllll|}
\hline Factors & \multicolumn{3}{l}{ Univariate COX regression } & \multicolumn{3}{c|}{ Multivariate COX regression } \\
& HR & $95 \% \mathrm{Cl}$ & $\mathrm{P}$ & $\mathrm{HR}$ & $95 \% \mathrm{Cl}$ & $\mathrm{P}$ \\
\hline Sex & $\mathbf{3 . 8 2 6}$ & $2.806-5.215$ & 0 & $\mathbf{4 . 9 1 5}$ & $3.578-6.751$ & 0 \\
\hline Age & $\mathbf{1 . 2 2 2}$ & $1.016-1.472$ & 0.03 & 1.023 & $0.863-1.212$ & 0.796 \\
\hline Degree & $\mathbf{1 . 9 7 4}$ & $1.675-2.326$ & 0 & $\mathbf{1 . 9 6 1}$ & $1.651-2.331$ & 0 \\
\hline Stage_T & $\mathbf{0 . 9 1 6}$ & $0.813-1.032$ & 0.15 & 0.936 & $0.825-1.061$ & 0.299 \\
\hline Stage_N & $\mathbf{0 . 5 5 2}$ & $0.466-0.653$ & 0 & $\mathbf{0 . 5 3 2}$ & $0.445-0.636$ & 0 \\
\hline Stage_M & $\mathbf{0 . 0 4 6}$ & $0.003-0.831$ & 0.04 & 0 & $0-0.001$ & 0.946 \\
\hline
\end{tabular}

Bold denotes statistical significance.

Adjusted for $x x, x x x, x x x$

Table. 5 Baseline data of validation data of nasopharyngeal carcinoma 


\begin{tabular}{|c|c|c|c|c|}
\hline & & code & $\mathrm{n}$ & $\%$ \\
\hline \multirow[t]{2}{*}{ Sex } & Male & 1 & 70 & 72.9 \\
\hline & Female & 2 & 26 & 27.1 \\
\hline \multirow[t]{5}{*}{ Age } & $<20$ & 1 & 1 & 1 \\
\hline & $>=20,<40$ & 2 & 13 & 13.5 \\
\hline & $>=40,<60$ & 3 & 55 & 57.3 \\
\hline & $>=60,<80$ & 4 & 26 & 27.1 \\
\hline & $>=80$ & 5 & 1 & 1 \\
\hline \multirow[t]{4}{*}{ Degree } & No differentiation & 0 & 7 & 7.3 \\
\hline & Poorly differentiated & 1 & 26 & 27.1 \\
\hline & Moderate differentiation & 2 & 17 & 17.7 \\
\hline & Highly differentiated & 3 & 46 & 47.9 \\
\hline \multirow[t]{5}{*}{ Stage_T } & $\mathrm{T} 1$ & 1 & 9 & 9.4 \\
\hline & $\mathrm{T} 2$ & 2 & 27 & 28.1 \\
\hline & T3 & 3 & 21 & 21.9 \\
\hline & $\mathrm{T} 4$ & 4 & 13 & 13.5 \\
\hline & $\mathrm{TX}$ & 5 & 26 & 27.1 \\
\hline \multirow[t]{5}{*}{ Stage_N } & No & 0 & 5 & 5.2 \\
\hline & N1 & 1 & 15 & 15.6 \\
\hline & N2 & 2 & 44 & 45.8 \\
\hline & N3 & 3 & 7 & 7.3 \\
\hline & $N X$ & 4 & 25 & 26.0 \\
\hline \multirow[t]{2}{*}{ Stage_M } & MO & 0 & 67 & 69.8 \\
\hline & $M x$ & 1 & 29 & 30.2 \\
\hline \multirow[t]{2}{*}{ Metastasis } & Yes & 1 & 53 & 55.2 \\
\hline & No & 0 & 43 & 44.8 \\
\hline
\end{tabular}

Figures 


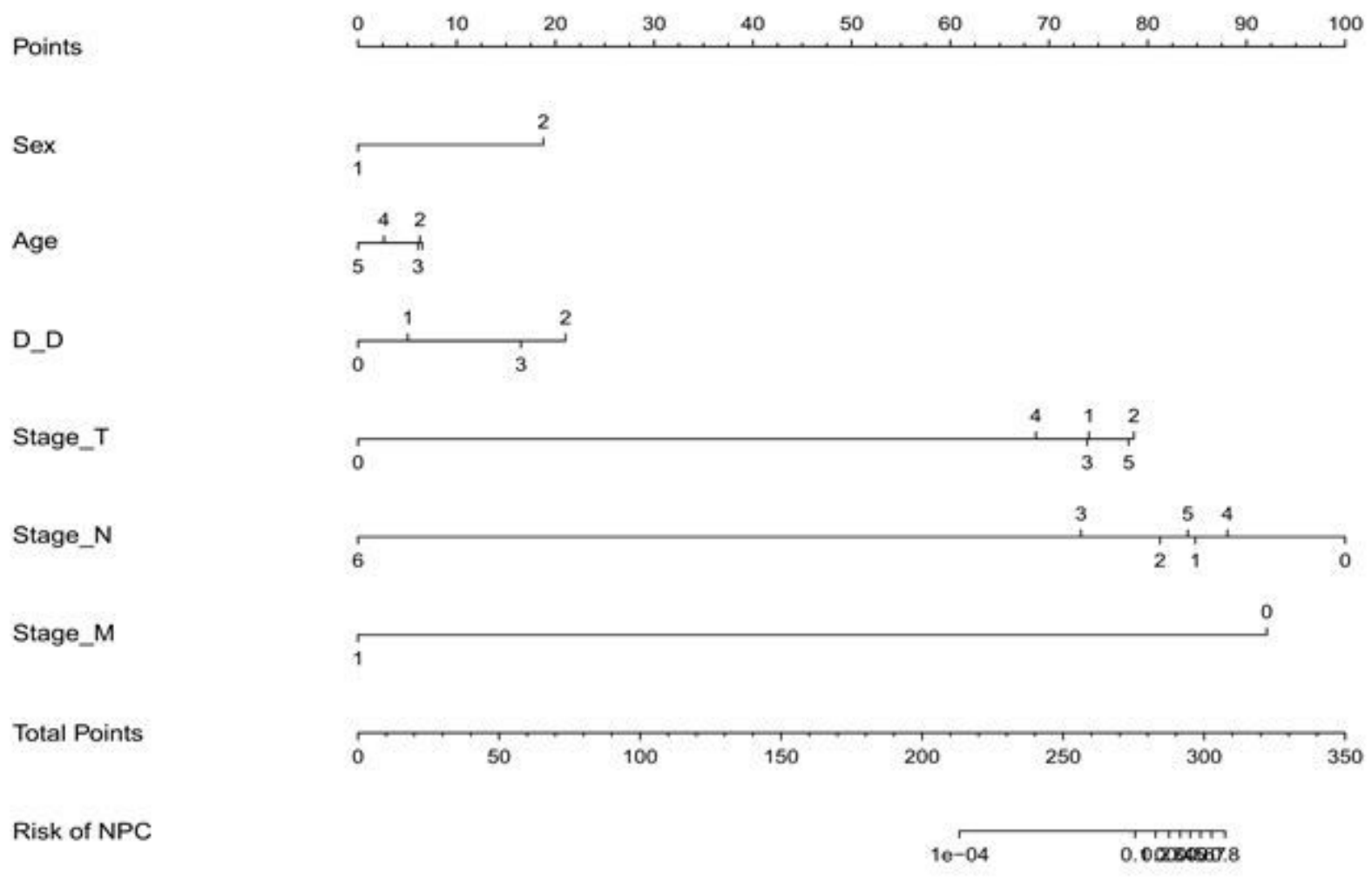

\section{Figure 1}

Nasopharyngeal cancer risk nomogram prediction model

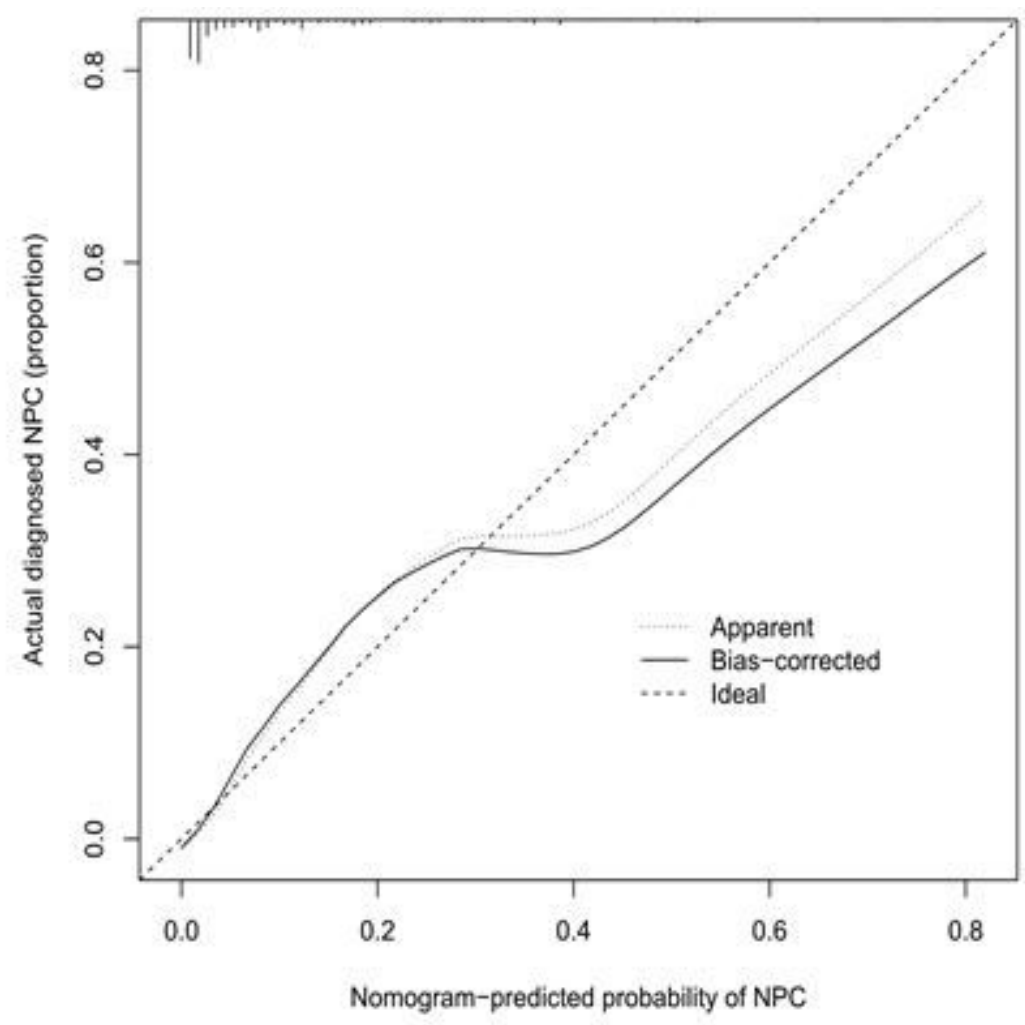

Figure 2 
Calibration curve of nasopharyngeal carcinoma risk prediction model

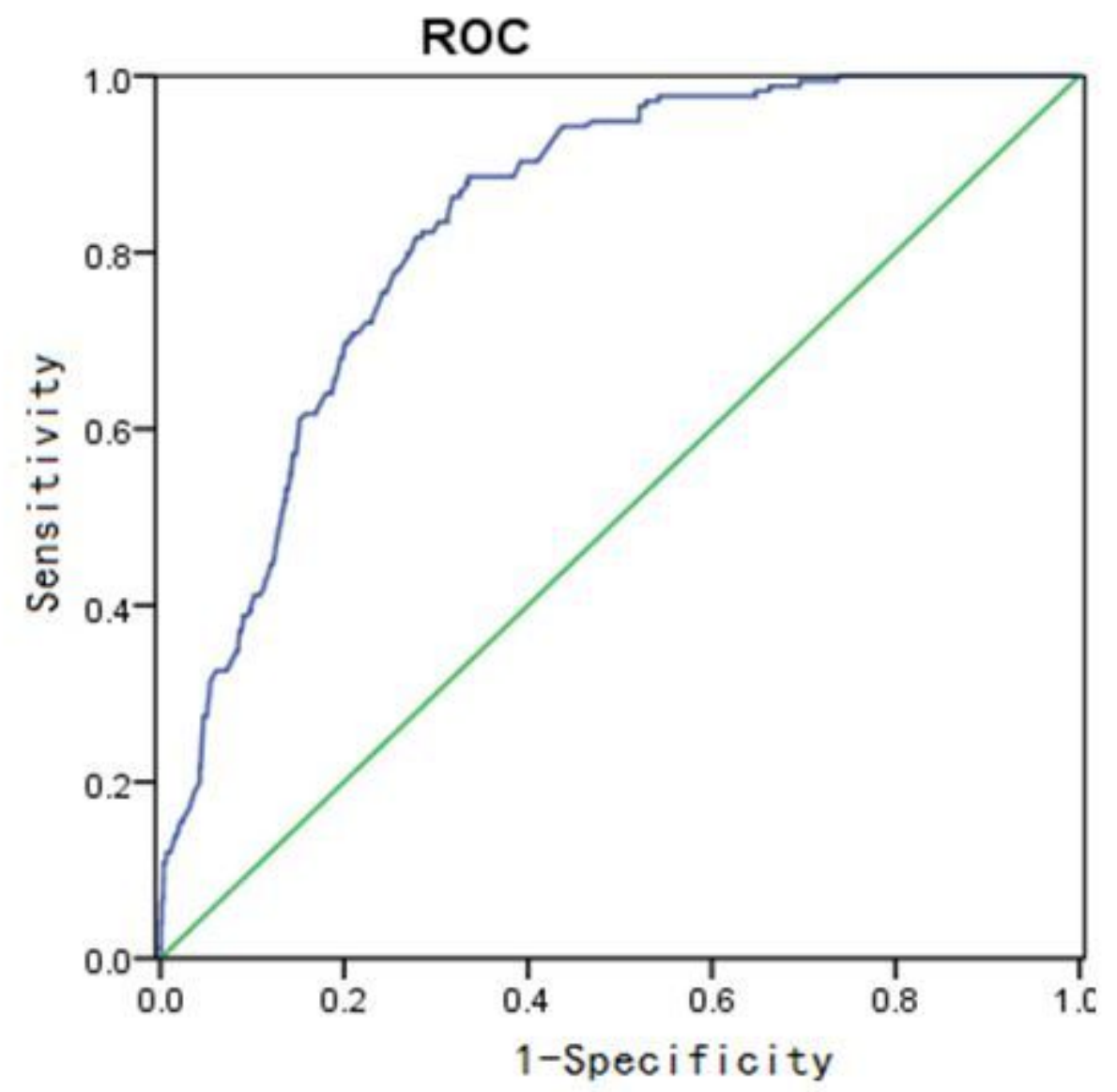

Figure 3

ROC curve of nasopharyngeal carcinoma in 3.SEER database 


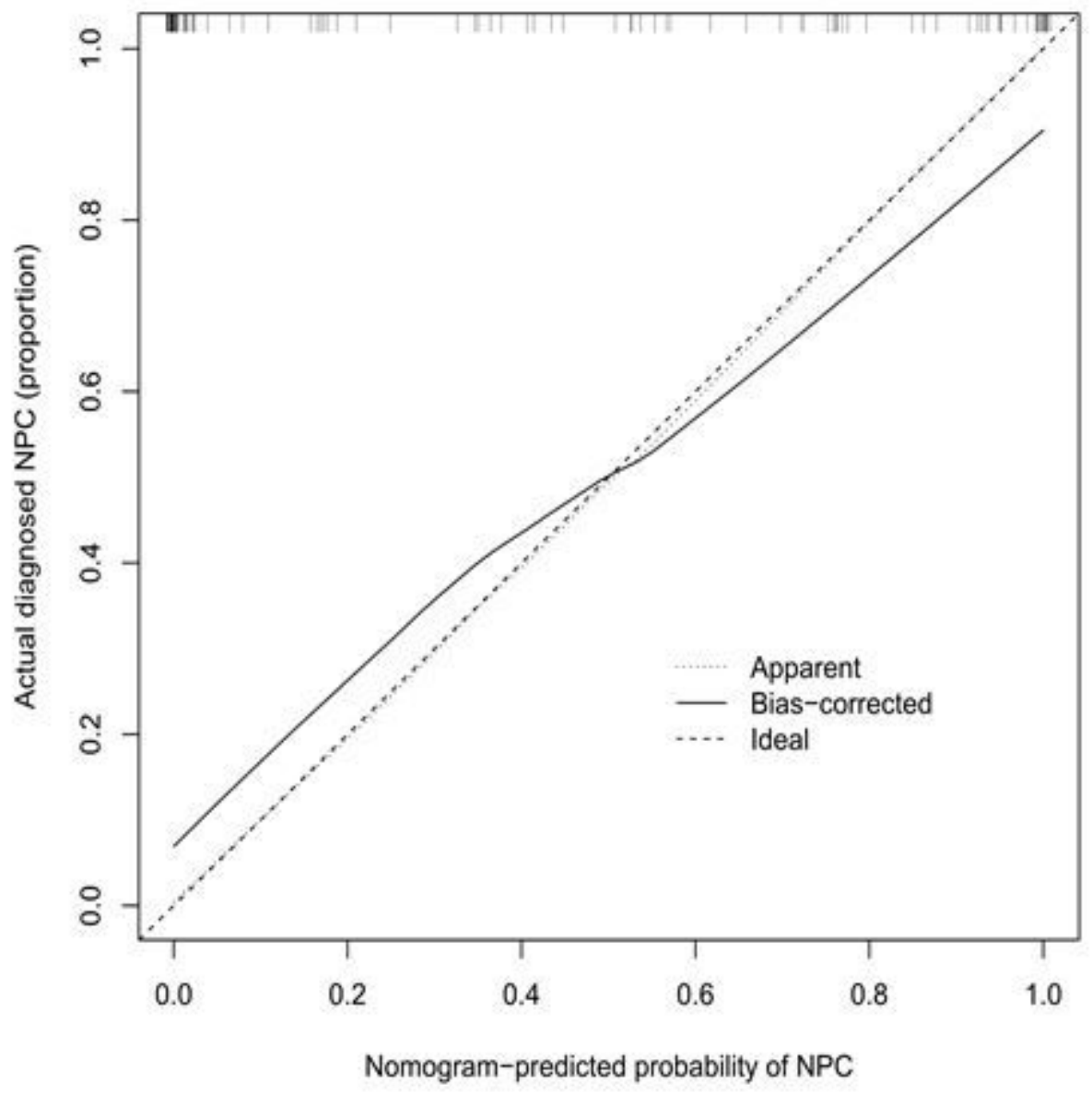

Figure 4

Calibration curve of nasopharyngeal carcinoma risk prediction model for clinical validation data 


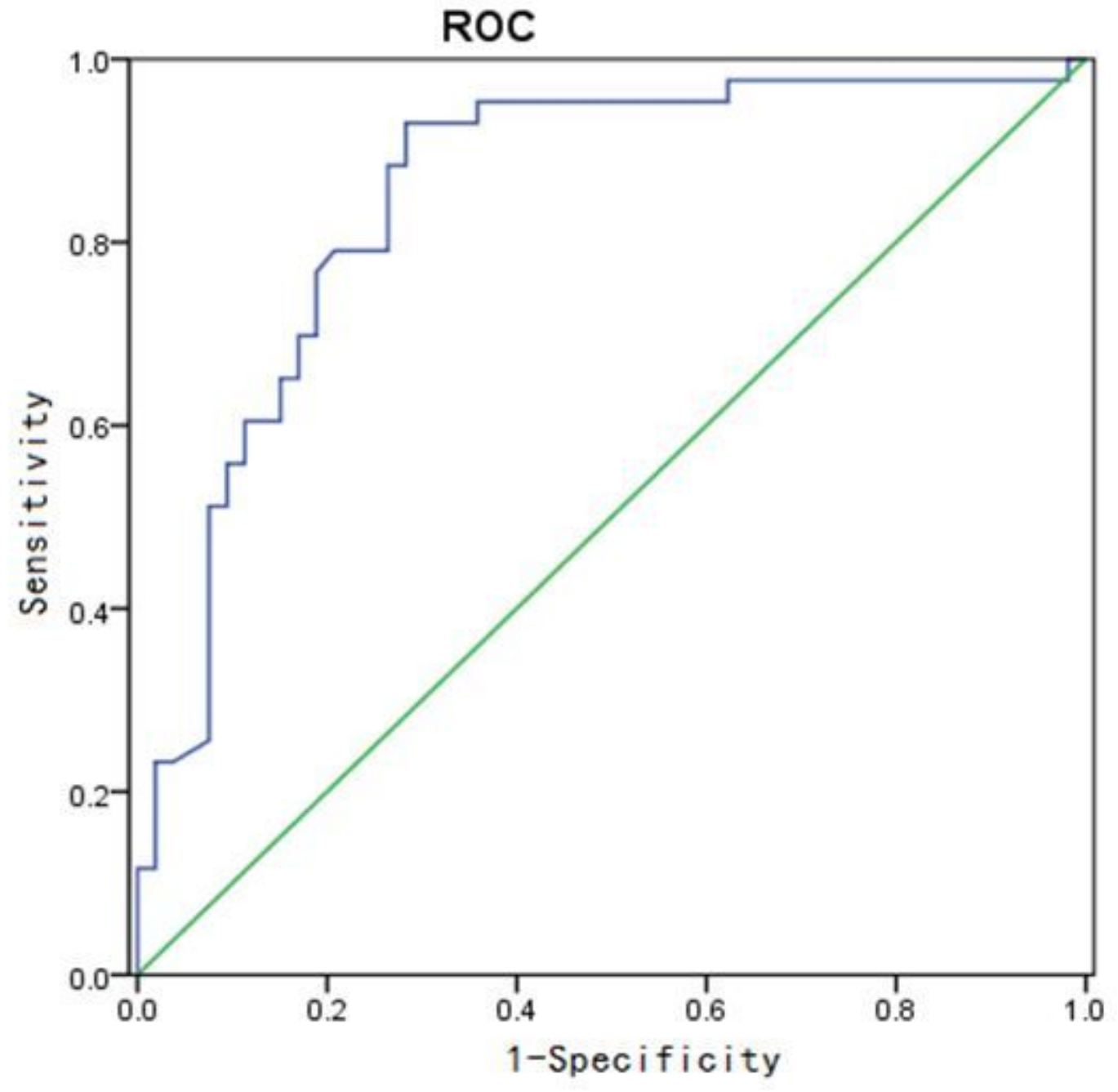

Figure 5

ROC curve of nasopharyngeal carcinoma with clinical validation data 